\title{
Prospecting endophytic fungal assemblage of Digitalis lanata Ehrh. (foxglove) as a novel source of digoxin: a cardiac glycoside
}

\author{
Sanjana Kaul • Maroof Ahmed · Khalid Zargar • \\ Pooja Sharma $\cdot$ Manoj K. Dhar
}

Received: 27 August 2012/ Accepted: 3 December 2012/Published online: 15 December 2012

(C) The Author(s) 2012. This article is published with open access at Springerlink.com

\begin{abstract}
Endophytes, the chemical synthesizers inside plants, are the microorganisms having mutualistic relationship with the host plant. They can be used by plants for defense in addition to the production of a wide variety of beneficial bioactive secondary metabolites. There are reports that microbial endophytes mimic the bioactive compounds as produced by the plant itself thus making them a promising source of novel compounds. During the present study, endophytes were isolated from the symptomless leaves and stem of the angiosperm, Digitalis lanata (foxglove). Digitalis lanata belongs to the family Plantaginaceae and is an important medicinal plant known for the production of an important glycoside, digoxin having valuable medicinal importance. Glycosides from Digitalis have been reported to be cardiotonic and are widely used in the treatment of various heart conditions namely atrial fibrillation, atrial flutter, heart failure, etc. Endophytic fungi were isolated from Digitalis to screen them for such glycosides as have been found in the plant itself. A total of 35 fungal endophytes were isolated and screened for the production of secondary metabolites. After preliminary analysis by thin layer chromatography for the presence of bioactive compounds, crude extracts of five fungal cultures were selected for HPLC. HPLC chromatograms revealed the production of glycoside digoxin from the five selected endophytic cultures, thus providing a novel, alternative and eco-friendly source for the production of such a pharmaceutically important and valuable drug.
\end{abstract}

S. Kaul $(\varangle) \cdot$ M. Ahmed · K. Zargar · P. Sharma · M. K. Dhar School of Biotechnology, University of Jammu, Jammu 180006, India

e-mail: sanrozie@ rediffmail.com
Keywords Endophytes - Secondary metabolites . Glycosides · Digitalis $\cdot$ Digoxin $\cdot$ HPLC

\section{Introduction}

Plants have been known to act as host to various endophytic microorganisms known to produce plethora of substances of potential use to modern medicine, agriculture and pharmaceutical industry (Owen and Hundley 2004). Endophytes usually have a mutualistic relationship with that of the host and are responsible for the adaptation of plants to abiotic stress such as drought, light, metals and biotic stress like herbivory, insects and pathogens (Arnold et al. 2003; Carroll 1988; Clay 1988; Faeth 2002). Endophytic fungi have been found in a diverse array of plant species examined so far including algae, mosses, conifers, angiosperms, and gymnosperms (U'ren et al. 2010). Endophytic fungi inhabiting the foliage of woody plants are far less studied than endophytes of grasses (Arnold and Herre 2003). Endophytes include fungi, bacteria and actinomycetes, although the most frequently isolated endophytes are the fungi. Endophytic fungi are considered as an outstanding source of bioactive natural products because there are many occupying millions of unique biological niches and growing in different types of environment (Strobel and Daisy 2003). Plants infected with fungal endophytes are often healthier than endophyte free ones (Waller et al. 2005). This effect may be due to the production of phytohormones such as indole 3-acetic acid (IAA), cytokines and plant growth promoting substances (Tan and Zou 2001). There are also quite a large number of biologically active compounds which have been isolated from endophytic fungi e.g., novel compounds like anticancer drugs, antibiotics and immunosuppressive compounds (Strobel and Daisy 2003). Various 
bioactive natural products derived from endophytic fungi belong to diverse structural groups including glycosides, flavonoids, terpenoids, steroids, xanthones, chinones, phenols, isocoumarins, benzopyrones, tetralones, etc. (Tan and Zou 2001). The presence of endophytes in plants stimulates the production of secondary metabolites in plants with a diverse range of biological activities (Petrini et al. 2006). Some of the endophytic microorganisms have been found to produce the same secondary metabolites as that of the plant thus making them a promising source of novel compounds. For example, the discovery of the world's first billion dollar anti-cancer drug paclitaxel (taxol) from Pestalotiopsis microspora, an endophytic fungus that colonizes the Himalayan yew tree Taxus wallichiana (Strobel et al. 1996; Gangadevi et al. 2008); Muscodor albus produces volatile compounds to inhibit and kill a wide spectrum of fungi and bacteria (Strobel et al. 2001); an anticancer compound camptothecin has been reported from Fusarium solani endophytic in Camptotheca acuminata (Kusari et al. 2009). Likewise, a number of anticancer compounds have been isolated from fungal endophytes (Kharwar et al. 2011). An insect repellent, azadirachtin (triterpenoid) has been reported from the endophytic fungus Eupenicillium parvum inhabiting Azadirachta indica. (Kusari et al. 2012a). Wang et al. (2012) isolated new antimicrobial compounds produced by Phoma sp. endophytic in Arisaema erubescens. Other important secondary metabolites isolated from endophytes include ergoflavin, griseofulvin, hypericin, citrinin, pestacin, methyl eugenol, 2-phenyl ethanol, etc. (Joseph and Priya 2011; Kaul et al. 2008; Wani et al. 2010). Kharwar et al. (2012) studied the diversity, distribution and community structure of endophytic fungi from Cinnamomum camphora and also evaluated their antimicrobial potential. Even much more significant research has been done on various aspects of endophytic fungi including diversity, mutualistic paradigms, chemical ecology, production of bioactive metabolites and various other industrial applications (Kaul et al. 2012; Kusari et al. 2012b, c, d; Kusari and Spiteller 2011). Thus, it becomes obvious that an enormous potential for new drug discovery in this field holds exciting promise. This is evidenced by the discovery of a wide range of products and microorganisms that present potential for new drug discovery and consequently, it motivated us to investigate the secondary metabolism of endophytic fungi in a medicinal plant with an aim to find the fungal strain able to produce structurally novel and biologically active secondary metabolites.

Digitalis (foxglove), an ornamental angiosperm of about 20 species of herbaceous perennials, shrubs and biennials has been reported to produce cardiotonic drugs and is widely used in the treatment of various heart conditions namely atrial fibrillation, atrial flutter and, sometimes heart failure that cannot be controlled by other medications. It is a powerful diuretic and provides a valuable remedy in dropsy. It has also been employed in the treatment of internal hemorrhage, inflammatory diseases, delirium tremens, epilepsy, acute mania and various other diseases with real or supposed benefit. It is used to increase cardiac contractility (it is a positive inotrope) and as an antiarrhythmic agent to control the heart rate, particularly in the irregular (and often fast) atrial fibrillation (Ahmed et al. 2012; Fatima et al. 2009).

In the present study, efforts have been made to isolate endophytic fungi inhabiting Digitalis lanata. The isolated endophytic fungal assemblage was processed and evaluated for the production of compounds particularly glycosides similar to those present in the plant itself.

\section{Materials and methods}

\section{Source of endophytic fungi}

The medicinal plant i.e., Digitalis lanata selected for the present study was collected from its natural habitat, Gulmarg (altitude 2,730 m; District Baramulla) Jammu and Kashmir State, India. The samples of the plant material were collected and brought to laboratory in pre-sterilized polythene bags and kept at $4{ }^{\circ} \mathrm{C}$ till immediate processing.

\section{Isolation of endophytic fungi}

Endophytic fungal isolation was carried out under aseptic conditions. Different parts of the wooly foxglove (Digitalis lanata) such as stem cuttings and leaves were used for the isolation of fungal endophytes (Bacon 1988; Ahmed et al. 2012). The collected plant material used for the isolation was first surface sterilized following the method of Santos et al. (2003) with slight modifications. Plant material was first cleaned by washing several times under running tap water and then cut into small segments. Surface sterilization was performed by sequentially rinsing the plant material with $70 \%$ ethanol $\left(\mathrm{C}_{2} \mathrm{H}_{5} \mathrm{OH}\right)$ for $30 \mathrm{~s}$, then with $0.01 \%$ mercuric chloride $\left(\mathrm{HgCl}_{2}\right)$ for $5 \mathrm{~min}$ followed by $0.5 \%$ working concentration of available sodium hypochlorite $4 \%$ solution $(\mathrm{NaOCl})$ for 2-3 min and finally with sterile distilled water $2-3$ times. It was then dried in between the folds of sterile filter paper. After proper drying, leaves and stem were cut into smaller segments, each segment was placed in a Petri dish plated with potato dextrose agar (PDA) medium supplemented with chloramphenicol $(100 \mathrm{mg} / \mathrm{ml})$. A total of 120 explants were inoculated on 20 PDA-plates (6 explants per plate). All the plates were incubated at $26{ }^{\circ} \mathrm{C}$ to promote fungal growth and sporulation. The plates were regularly monitored for any mycelial growth. On observing the mycelial growth 
sub-culturing of the fungi was done. Each fungal culture was checked for purity and transferred to another agar plate using the hyphal tips. The purified fungal isolates were transferred separately to PDA slants, accessioned and maintained at $4{ }^{\circ} \mathrm{C}$. Appropriate controls were also set up with media-plated plates without any material.

\section{Extraction of secondary metabolites}

Secondary metabolites were extracted from the plant material as well as from the fungal isolates.

\section{Plant material}

Extraction of secondary metabolites was done by Soxhlet extraction method. Leaves of Digitalis lanata were properly washed, air dried and ground to fine powder. Ten grams of this powder was placed in Soxhlet extractor and the assembly was run using hexane for $6-8 \mathrm{~h}$. After this, solvent was changed and the Soxhlet was run with $50 \%$ methanol $\left(\mathrm{CH}_{3} \mathrm{OH}\right)$ for 18-20 h. Crude extract so obtained was concentrated in a water bath and then hydrolyzed in $10 \% \mathrm{HCl}$ for $1 \mathrm{~h}$. The contents were then subsequently separated with chloroform in a separating funnel. Chloroform fractions were collected, concentrated and checked on preparative TLC plates.

\section{Selected endophytic fungal isolates}

Extraction of secondary metabolites from fungal endophytes was done by Soxhlet extraction method. A total of 35 fungal endophytes were isolated and screened for the production of secondary metabolites. Freshly grown fungal isolates were cultured in $150 \mathrm{ml}$ of potato dextrose production medium in a $250 \mathrm{ml}$ conical flask. The flasks were incubated at $27{ }^{\circ} \mathrm{C}$ in shaking conditions at $140 \mathrm{rpm}$. Maximum growth and optimum production were evaluated on the 12th day of incubation. The protocol was standardized for isolation of secondary metabolites from fungal endophytes. The fungal biomass was then filtered through muslin cloth and allowed to dry on a filter paper. The dried fungal biomass was then extracted with n-hexane in the Soxhlet extractor assembly for 8-10 h. After extraction with n-hexane the Soxhlet was run with $50 \%$ methanol for complete extraction of metabolites for 18-20 h from fungal biomass. The extract was then concentrated in a water bath followed by hydrolysis with $10 \% \mathrm{HCl}$. Separation was carried out with chloroform in a separating funnel. Chloroform fractions were collected, concentrated and checked by thin layer chromatography (TLC).

TLC of all the samples was performed on silica gel plates with the solvent system ethyl-acetate:methanol:water in the ratio of $(81: 11: 8) \mathrm{v} / \mathrm{v}$. The compounds were detected by spraying $4 \%$ sulfuric acid as the developing reagent onto the TLC plates. The compounds appeared as specific spots after baking the TLC plates for 10 min at $110{ }^{\circ} \mathrm{C}$.

High performance liquid chromatography (HPLC) analysis

HPLC was performed to confirm and purify the compounds in the samples. The HPLC system used in the present study consisted of an Agilent series 1100 equipped with a binary pump, an auto sampler an automatic degasser, an automatic thermostatic column oven, diode array detector and a computer with chemstation software for data analysis. The HPLC conditions used were as follows: Column used Merck RP-18 $(125 \mathrm{~mm} \times 4.6 \mathrm{~mm}: 5 \mu \mathrm{m})$, mobile phase (80:20) acetonitrile:water, flow rate $100 \mu \mathrm{l} / \mathrm{min}$, and wavelength $210 \mathrm{~nm}$.

\section{Results and discussion}

A total of 35 fungal endophytes were isolated from asymptomatic parts such as leaves and stem of Digitalis lanata. A total of 120 explants of both leaf and stem segments were inoculated on 20 PDA-plates (6-explants per plate). Nineteen fungal endophytes from leaves and sixteen fungal endophytes from the stem were isolated. The isolated endophytic fungi were then identified on the basis of morphological characters such as growth pattern, hyphae, color of colony, surface texture, margin character and characteristics of the spore (Barnett and Hunter 1956). It was found that among the fungal assemblage, frequency of Alternaria, Penicillium and Aspergillus species was maximum when compared to other genera, while few of them could not be identified due to lack of either sporulation or other significant characters of identification. Depending on the frequency of the species, they were taken up for further studies. The isolated fungal endophytes were further studied for glycoside production. They were subjected to liquid shake fermentation for the production of secondary metabolites. Secondary metabolites from endophytes have been reported to include growth hormones, antimicrobial or anticancer substances (Petrini et al. 1992; Huang et al. 2001). There are reports on secondary metabolites being potential drugs for treatment of newly developing diseases in humans and also plants and animals (Strobel and Daisy 2003). Endophytes from the medicinal plants are a treasure hunt for the production of bioactive metabolites (Kaul et al. 2012).

In our study, we used the plant extract along with the standard for comparative analysis. Thin layer chromatography screening revealed that only five endophytic isolates

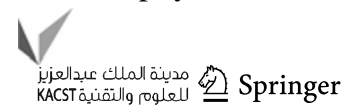



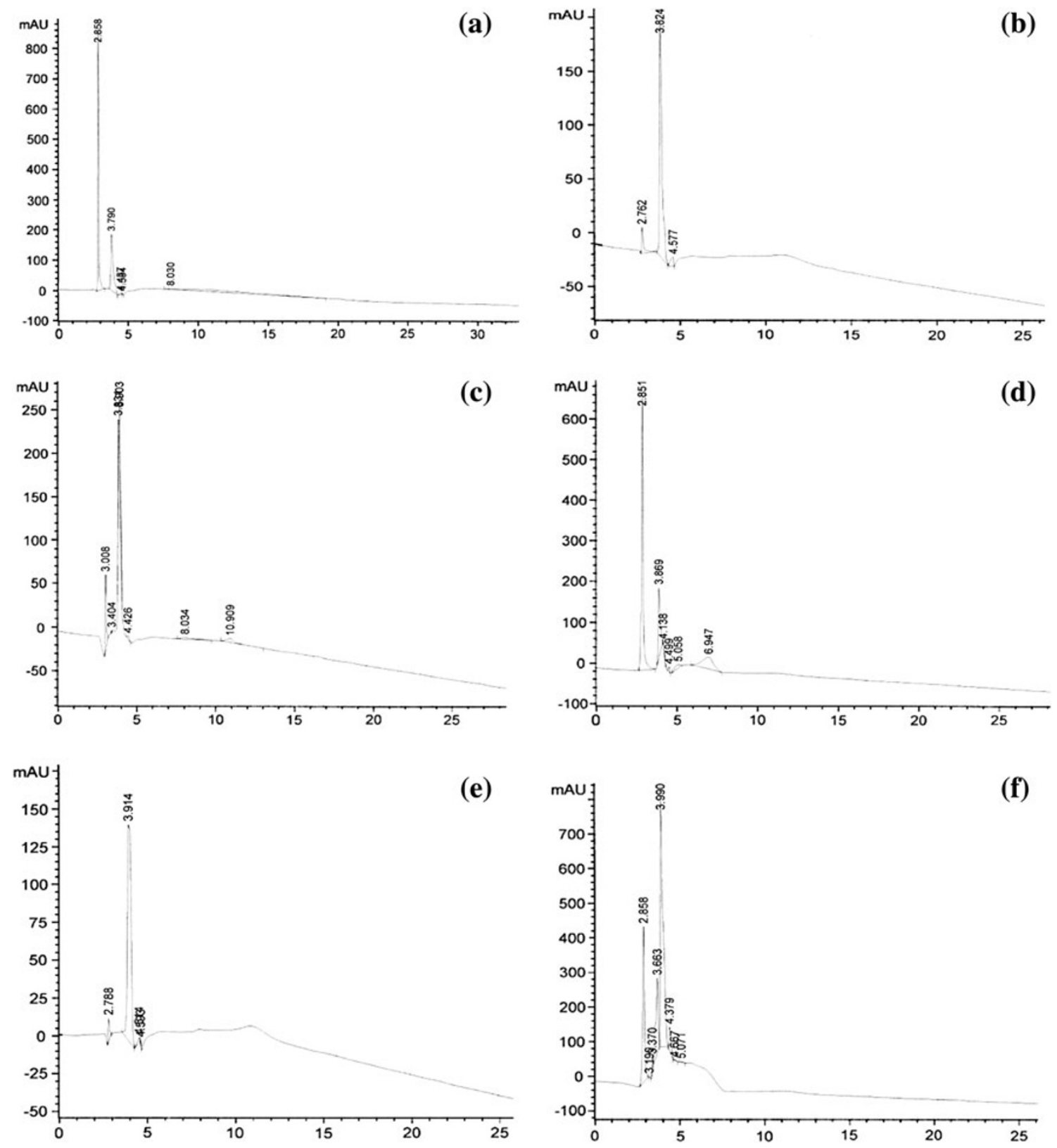

Fig. 1 HPLC-chromatograms of plant and endophytic fungal extracts exhibiting various peaks. a Chromatogram of the crude plant extract (Digitalis lanata) indicating various peaks. Peak at the retention time of $3.790 \mathrm{~min}$ corresponds to digoxin. b Chromatogram of the accession DL-3 indicating various peaks. Peak at the retention time of $3.824 \mathrm{~min}$ corresponds to digoxin. c Chromatogram of the accession DL-12 indicating various peaks. Peak at the retention time

accessioned as DL-3, DL-11, DL-12, DS-20 and DS-22 produced the spots corresponding to the relevant spot of the standard and plant extract. Thus, out of the total fungal assemblage consisting of 35 isolated endophytic fungi, only five potent isolates were selected for HPLC-analysis after the preliminary screening by TLC.

From the chromatograms, it could be observed that the plant extract (Fig. 1a) along with the endophytic fungal

of $3.831 \mathrm{~min}$ corresponds to digoxin. d Chromatogram of the accession DS-20 indicating various peaks. Peak at the retention time of $3.869 \mathrm{~min}$ corresponds to digoxin. e Chromatogram of the accession DL-11 indicating various peaks. Peak at the retention time of $3.914 \mathrm{~min}$ corresponds to digoxin. f Chromatogram of the accession DS-22 indicating various peaks. Peak at the retention time of $3.663 \mathrm{~min}$ corresponds to digoxin

accessions (Fig. 1b-f) eluted different compounds at different retention times as are exhibited by various peaks. Here it could be seen that fungal endophytes mimic some of the compounds as produced by the plant itself. This was revealed by retention times which exactly/nearly correspond with the retention times of various peaks shown by the plant extract. Most prominent peaks from all the fungal accessions at the retention times of $3.824,3.914,3.831$, 
3.869 and $3.663 \mathrm{~min}$, approximately, correspond to one of the compounds eluted by the plant extract at a retention time of 3.790 min which was also the retention time of standard (digoxin) under the same HPLC conditions. Thus, it can be concluded that the main chemical constituent of Digitalis lanata i.e., the glycosidic compound digoxin was produced by the five fungal endophytes under investigation that were isolated from this plant. Apart from this, some other compounds from the fungal isolates also correspond with the rest of the compounds eluted from the plant extract i.e., the compound from isolate DS-22 at the retention time of 2.858 min exactly corresponds to one of the peaks from the plant extract and nearly with one of the peaks from all other fungal isolates. Likewise, rest of the peaks from the plant extract nearly corresponds to some of the peaks from the endophytic fungal accessions. Thereby, confirming that the fungal endophytes do mimic the bioactive compounds as produced by the plant itself (digoxin in this case). Thus, providing a new hope for the production of novel metabolites through alternative means that even being ecofriendly.

Natural products from fungal endophytes have a broad spectrum of biological activity and they can be grouped into several categories including; alkaloids, steroids, terpenoids, isocoumarins, quinones, phenyl propanoids and lignans, aliphatic metabolites, lactones, etc. (Zhang et al. 2006). Puri et al. (2005), isolated a novel Camptothecin producing endophytic fungus Entrophosphora inferquens from an important Indian medicinal plant Nothapodytes foetida. E. inferquens synthesizes camptothecin which showed potential immunomodulatory activity. Chen et al. (2007) isolated an endophytic fungus Pencillium thomi from the roots of Bruguiera gymnorhiza. The separation of endophytic fungus from the root led to the isolation of a new compound $4^{\prime}, 5$ dihydroxy-2,3 dimethoxy 4(-hydroxy propyl)-biphenyl along with 11 known compounds. Their effects against three human cell lines were investigated. In the series of useful bioactive compounds from plants, two important compounds are digoxin and digitoxin. These are plant glycosides (Cardiac glycosides) which are widely used in medicine. These glycosides are produced from the medicinal plant Digitalis lanata. Digitalis glycosides consist of a steroid nucleus, an unsaturated lactone ring and a sugar stuck on to the lactone ring. The sugar determines solubility, half life, etc. The main chemical constituent of Digitalis lanata is Digoxin. Digoxin has an extra $\mathrm{OH}$ group making it more polar (less GI absorption, reduced protein binding and increased urinary excretion related to digitoxin). Digoxin is eliminated chiefly by the liver/kidneys. It exists as odorless white crystals that melt with decomposition above $230{ }^{\circ} \mathrm{C}$. The drug is practically insoluble in water and in ether; slightly soluble in diluted (50\%) alcohol and in chloroform; and freely soluble in pyridine.
Kwon et al. (2010) detected cardiac glycosides from the leaves of Digitalis by reversed phase HPLC-pulsed amperometric detection. Further, the content of the cardiotonic glycosides from Digitalis was determined by Alonso et al. (2009). A microbial source for the production of this glycoside would really be beneficial.

Therefore, keeping in view the pharmaceutical applications of digoxin, these glycoside producing potent strains of endophytic fungi comprising a fungal assemblage could be further worked out on a pilot scale for process optimization and scale-up studies. This study signifies a novel microbial source for the digoxin-like glycosides.

\section{Conclusions}

Endophytic fungi have recently received more attention as they can sometimes produce bioactive compounds analogous to their hosts. The mechanism is still not clear but it is assumed that the genes which control the synthesis of bioactive compounds might be transferred between endophytic fungi and the host during long time symbiosis. We can say that the endophytic fungi could be a reliable source for pharmaceutically and industrially important compounds that can be used in the treatment of various life-threatening diseases along with various industrial applications. Applications of endophytes in pharmaceutical industries include cost effective drug production, endophytes as drug source help us to conserve biodiversity and drug resistance as they are an alternate source of drugs.

Acknowledgments The authors are grateful to Department of Biotechnology, Govt. of India and Council of Scientific and Industrial Research, New Delhi for facilities and funding (CSIR No. 38(1281)/ 11/EMR-II).

Conflict of interest The authors declare that they have no conflict of interest

Open Access This article is distributed under the terms of the Creative Commons Attribution License which permits any use, distribution, and reproduction in any medium, provided the original author(s) and the source are credited.

\section{References}

Ahmed M, Hussain M, Dhar MK, Kaul S (2012) Isolation of microbial endophytes from some ethnomedicinal plants of Jammu and Kashmir. J Nat Prod Plant Resour 2(2):215-220

Alonso et al (2009) Cardiotonic glycosides from biomass of Digitalis purpurea $\mathrm{L}$. cultured in temporary immersion systems. Plant Cell Tiss Organ Cult 99:151-156

Arnold AE, Herre EA (2003) Canopy cover and leaf age affect colonization by tropical fungal endophytes: ecological pattern and process in Theobroma cacao (Malvaceae). Mycologia 95(3):388-398 
Arnold AE, Mejia LC, Kyllo D, Rojas EI, Maynard Z, Robbins N, Herre EA (2003) Fungal endophytes limit pathogen damage in tropical trees. Proc Natl Acad Sci USA 100(26):15649-15654

Bacon CW (1988) Procedure for isolating the endophyte from tall fescue and screening isolates for ergot alkaloids. Appl Environ Microbiol 54(11):2615-2618

Barnett HL, Hunter BB (1956) Illustrated genera of imperfect fungi, 2nd edn. Burgess publishing, Minneapolis, p 218

Carroll GC (1988) Fungal endophytes in stems and leaves: from latent pathogen to mutualistic symbionts. Ecology 69:2-9

Chen et al (2007) The metabolites of a mangrove endophytic fungus, Penicillium thomi. J Asian Nat Prod Res 9(2):59-164

Clay K (1988) Fungal endophyte of grasses a defence mutualisms between plant and fungi. Ecology 69:10-16

Faeth SH (2002) Are endophytic fungi defensive plant mutualists. Oikos 98(1):25-36

Fatima Z, Mujib A, Fatima S, Arshi A, Umar S (2009) Callus induction, biomass growth, and plant regeneration in Digitalis lanata Ehrh.: influence of plant growth regulators and carbohydrates. Turk J Bot 33:393-405

Gangadevi V, Murugan M, Muthumary M (2008) Taxol determination from Pestalotiopsis pauciseta, a fungal endophyte of a medicinal plant. Chin J Biotechnol 24:1433-1438

Huang Y, Wang J, Li G, Zhang Z, Su W (2001) Antitumor and Antifungal activities in endophytic fungi isolated from pharmaceutical plants Taxus mairei, Cephalataxus fortunei and Torreya grandis. FEMS Immunol Med Microbiol 31(2):163-167

Joseph B, Priya RM (2011) Bioactive compounds from endophytes and their potential in pharmaceutical effect. Am J Biochem Mol Biol 1(3):291-309

Kaul S, Wani M, Dhar KL, Dhar MK (2008) Production and GC-MS trace analysis of methyl eugenol from endophytic isolate of Alternaria from rose. Ann Microbiol 58(3):443-445

Kaul S, Gupta S, Ahmed M, Dhar MK (2012) Endophytic fungi from medicinal plants: a treasure hunt for bioactive metabolites. Phytochem Rev. doi:10.1007/s11101-012-9260-6

Kharwar RN, Mishra A, Gond SK, Stierle A, Stierle D (2011) Anticancer compounds derived from fungal endophytes: their importance and future challenges. Nat Prod Rep 28:1208-1228

Kharwar RN, Maurya AL, Verma VC, Kumar A, Gond SK, Mishra A (2012) Diversity and antimicrobial activity of endophytic fungal community isolated from medicinal plant Cinnamomum camphora. Proc Natl Acad Sci India sect B Biol Sci 82(4):557-565

Kusari et al (2009) An endophytic fungus from Camptotheca acuminata that produces camptothecin and analogues. J Nat Prod 72(1):2-7

Kusari S, Spiteller M (2011) Are we ready for industrial production of bioactive plant secondary metabolites utilizing endophytes? Nat Prod Rep 28:1203-1207

Kusari P, Kusari S, Spiteller M, Kayser O (2012a) Endophytic fungi harbored in Cannabis sativa L.: diversity and potential as biocontrol agents against host-plant specific phytopathogens. Fungal diversity. doi:10.1007/s13225-012-0216-3
Kusari S, Hertweck C, Spiteller M (2012b) Chemical ecology of endophytic fungi: origins of secondary metabolites. Chem Biol 19:792-798

Kusari S, Pandey SP, Spiteller M (2012c) Untapped mutualistic paradigms linking host plant and endophytic fungal production of similar bioactive secondary metabolites. Phytochemistry. doi: 10.1016/j.phytochem.2012.07.021

Kusari S, Verma VC, Lamshoft M, Spiteller M (2012d) An endophytic fungus from Azadirachta indica A. Juss. that produces azadirachtin. World J Microbiol Biotechnol 28:1287-1294

Kwon et al (2010) HPLC method validation for Digitalis and its analogue by pulsed amperometric detection. J Pharma Biomed Anal 54:217-221

Owen NL, Hundley N (2004) Endophytes-the chemical synthesizers inside plants. Sci Prog 87(2):79-99

Petrini O, Sieber TN, Toti L, Viret O (1992) Metabolite production and substrate utilization in endophytic fungi-Natural toxins. Ecology 1:185-196

Petrini O, Sieber TN, Toti L, Viret O (2006) Ecology, metabolite production, and substrate utilization in endophytic fungi. Nat Tox 1(3):185-196

Puri et al (2005) An endophytic fungus from Nothapodytes foetida that produces camptothecin. J Nat Prod 68(12):1717-1719

Santos RMG, Rodrigues-FoE Rocha WC, Teixeira MFS (2003) Endophytic fungi from Melia azadirchta. World J Microbiol Biotech 19:767-770

Strobel G, Daisy B (2003) Bioprospecting for microbial endophytes and their natural products. Microbiol Mol Biol Rev 67(4): 491-502

Strobel G et al (1996) Taxol from Pestalotiopsis microspora, an endophytic fungus of Taxus wallachiana. Microbiology 142: 435-440

Strobel G et al (2001) Volatile antimicrobials from Muscodor albus, a novel endophytic fungus. Microbiology 147:2943-2950

Tan RX, Zou WX (2001) Endophytes: a rich source of functional metabolites. Nat Prod Rep 18:448-459

U'ren JM, Lutzoni F, Miadlikowska J, Arnold AE (2010) Community analysis reveals close affinities between endophytic and endolichenic fungi in mosses and lichens. Microb Ecol 60:340-353

Waller et al (2005) The endophytic fungus Piriformospora indica reprograms barley to salt stress tolerance, disease resistance and higher yield. Proc Natl Acad Sci USA (PNAS) 102:13386-13391

Wang LW, Xu BG, Wang JY, Su ZZ, Lin FC, Zhang CL, Kubicek CP (2012) Bioactive metabolites from Phoma species, an endophytic fungus from the Chinese medicinal plant Arisaema erubescens. Appl Microbiol Biotechnol 93:1231-1239

Wani MA, Kaul S, Dhar MK, Dhar KL (2010) GC-MS analysis reveals production of 2-phenylethanol from Aspergillus niger endophytic in rose. J Basic Microbiol 50:110-114

Zhang HW, Song YC, Tan RX (2006) Biology and chemistry of endophytes. Nat Prod Rep 23:753-771 